\title{
Status of astronomy in Rwanda and volunteer work at Kigali Institute of Education (KIE)
}

\author{
M. Pović ${ }^{1}$, P. Nkundabakura ${ }^{2}$ and J. Uwamahoro ${ }^{2}$ \\ ${ }^{1}$ Instituto de Astrofísica de Andalucía (IAA-CSIC), Granada, Spain; email: mpovic@iaa.es \\ ${ }^{2}$ Kigali Institute of Education (KIE), Maths-Physics Department, Kigali, Rwanda \\ emails: nkundapheneas@yahoo.fr, uwamahorojean@yahoo.fr
}

\section{Status of Astronomy in Rwanda}

Until 2009, astronomy was undeveloped in Rwanda, without astronomy courses at universities and schools, astronomical facilities, or any outreach programmes. With the international year of astronomy in 2009, Dr. Pheneas Nkundabakura and Dr. Jean Uwamahoro from the KIE Maths-Physics department, both graduates from the South African NASSP Programme (http://www.star.ac.za), started a program of implementing the astronomical knowledge at schools and universities. During the same year 2009, IAU donated 100 galileoscopes for the secondary schools, and several astronomy workshops were organised for the teachers. IAU donated also 5 laptops to help students and lecturers to learn and use astronomy software. With this, KIE students have now a possibility to choose astronomy/space science for their undergraduate final year research projects. Moreover, there is an ongoing effort to look for further collaboration towards establishing the first astronomical facility (observatory) in the country.

\section{Advancing astronomy in Rwanda through volunteer work}

In 2012 Dr. Mirjana Pović spent one month at KIE as a visiting astronomer. During her stay, her activities consisted in giving public talks to students and lecturers, giving a short astronomy courses to students, supervising three students in their research in photometry and help students with linux and latex. Before going to Rwanda people from the IAA-CSIC in Spain recollected a number of scientific books, 2 projectors, 1 computer, and 1 external disc (containing lots of books of science and in particular of Astronomy/Space science in electronic format) for the KIE Maths-Physics department.

\section{Thoughts for way forward}

Education is one of the most powerful tools to fight against the poverty on the long time-scales. Therefore sharing the knowledge with the less developed countries can be one way of contributing to boost the worldwide development in education and in particular in the area of astronomy. In these regards, the Office of Astronomy for Development (http://www.astro4dev.org/) is one of the possibilities to promote this type of partnerships where using professional skills and astronomy as an entertaining and inspiring science, people can improve children's critical thinking, their motivation for learning, and increase the level of education and research at schools and universities. Moreover, in this era where much of the astronomical data can be accessed online, astronomers can assist in teaching various techniques and software for data reduction and analysis and hence, ensure that universities in undeveloped countries perform consistent and productive astronomy research locally. 